\title{
Cereal consumption and indicators of cardiovascular risk in adolescent girls
}

\author{
Debra L Franko ${ }^{1}$, Ann M Albertson ${ }^{2, *}$, Douglas R Thompson ${ }^{3}$ and Bruce A Barton ${ }^{3}$ \\ ${ }^{1}$ Department of Counseling and Applied Educational Psychology, Northeastern University, Boston, MA, USA: \\ ${ }^{2}$ Bell Institute of Health and Nutrition, General Mills Inc., 9000 Plymouth Avenue, North Minneapolis, \\ Minnesota, MN 55427, USA: ${ }^{3}$ Maryland Medical Research Institute, Baltimore, MD, USA
}

Submitted 25 March 2010: Accepted 1 June 2010: First published online 19 July 2010

\begin{abstract}
Objective: To examine the association between cereal consumption and cardiovascular risk factors including waist, height, total cholesterol, LDL cholesterol and HDL cholesterol in a sample of adolescent girls.

Design: Longitudinal study.

Setting: The study was conducted from 1987 to 1997 and data were collected at three study sites (University of California at Berkeley, University of Cincinnati and Westat Inc., Rockville, MD, USA). Mixed models were used to estimate the association between the number of days of eating cereal and these four outcome variables.

Subjects: Girls ( $n$ 2371) who participated in the 10-year National Heart, Lung, and Blood Growth and Health Study (NGHS) and completed a $3 \mathrm{~d}$ food diary in years $1-5$ and 7,8 and 10 .

Results: Adolescent girls who ate cereal more often had lower waist-to-height ratio $(P<0 \cdot 005)$, lower total cholesterol $(P<0 \cdot 05)$ and lower LDL cholesterol $(P<0 \cdot 05)$, taking into account sociodemographic variables, physical activity levels and total energy intake.

Conclusions: Findings suggest that cereal consumption is associated with markers of cardiovascular risk and that childhood patterns of consumption may influence the development of risk factors later in adolescence.
\end{abstract}

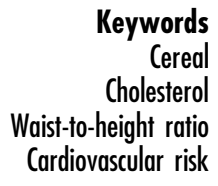

eywords

Cereal

Waist-to-height ratio Cardiovascular risk
Cereal is a popular breakfast choice for children. Children who consume cereal, relative to other breakfast foods, are less likely to be overweight or obese and evidence lower $\mathrm{BMI}^{(1-6)}$. One study of Greek children aged 12-17 years indicated that youth who ate cereal had 33\% lower probability of being overweight or obese, regardless of age, gender or degree of physical activity ${ }^{(3)}$. Williams ${ }^{(6)}$ reported that the lowest mean BMI and mean waist circumference were found in children (aged 1-12 years) who consumed cereal at breakfast when compared with those who ate other types of breakfasts or skipped breakfast.

Although weight is a useful indicator of health, it is not necessarily optimal. In fact, recent research has shown that waist-to-height ratio (WHtR) is a better predictor of health and disease outcomes than BMI, although most of these studies have been conducted with adults ${ }^{(7-10)}$. Notably, a recently published meta-analysis concluded that 'statistical evidence supports the superiority of .... WHtR over BMI, for detecting cardiovascular risk factors in both men and women, ${ }^{, 11)}$.

The advantage of WHtR as a discriminator for CVD risk has also been found in children, although the studies are fewer in number. Kahn et al. ${ }^{(12)}$ analysed data from 4- to 17-year-olds who participated in the Third National
Health and Nutrition Examination Survey (NHANES III) in a population-based comparison of BMI and WHtR to predict CVD risk. This large-scale study found that WHtR was a significantly better predictor of adverse cardiovascular risk factors than BMI. McCarthy and Ashwell ${ }^{(13)}$ reported that WHtR is more closely linked to childhood morbidity than BMI, and suggested it to be used as an 'additional or alternative measure to BMI' (p. 988) in studies of health.

A second useful indicator of CVD risk is cholesterol level $^{(14)}$. Elevated LDL cholesterol levels are a major risk factor for CVD and the need for management of high cholesterol levels in children was recently highlighted ${ }^{(15)}$. Because cholesterol levels can be altered by changes in eating habits, increasing our understanding of the relationship between dietary factors and cholesterol levels may have important implications for prevention. Although several studies have examined the relationship between cereal consumption and cholesterol levels in adults $^{(16)}$, very few have focused on children ${ }^{(17)}$. In one large study of 4-18-year-olds, Gibson ${ }^{(18)}$ reported that relative to low or average consumers, high consumers of breakfast cereals had lower total and LDL cholesterol. Nicklas et $a l .{ }^{(19)}$ provided evidence that serum lipid and 
lipoprotein levels continue to track from childhood into young adulthood, suggesting the need for preventive measures aimed at developing healthy lifestyles early in life. Thus, furthering our understanding of the relationship between health risk markers, such as WHtR and cholesterol, and dietary behaviour, such as cereal consumption, is important for both predicting and potentially managing early risk factors for CVD.

The goal of the current study was to examine associations between cereal consumption and WHtR as well as total LDL and HDL, cholesterol. Based on previous research, we hypothesized that greater cereal consumption would be associated with lower WHtR as well as lower total and LDL cholesterol.

\section{Experimental methods}

As previously reported, the National Heart, Lung, and Blood Growth and Health Study (NGHS) is a 10-year longitudinal study of 2379 girls who were 9 or 10 years old at study entry ${ }^{(20)}$. Participants (all female) were recruited from three study sites in the USA: University of California at Berkeley, University of Cincinnati/Cincinnati Children's Hospital Medical Center and Westat Inc./Group Health in Rockville, MD. The study protocol was approved by the Institutional Review Boards of all participating sites. All girls who entered the NGHS had assented and a parent or guardian consented to their participation.

\section{Procedures and measures}

Three-day food records that had been previously validated $^{(21)}$ were collected at visits (study years) $1-5$ and then again at years 7,8 and 10. Dietitians used ageappropriate materials to instruct girls to record all food and drink, type of meal (breakfast, snack, lunch, etc.) and time of intake for three consecutive days that included two weekdays and one weekend day. Dietitians rated each eating event reported in the food diaries as either a 'meal' or a 'snack'. Food records were coded and analysed for nutrient content ${ }^{(22)}$. Nutrient values were updated annually to reflect changes in the nutrient composition of individual foods. The predictor of interest was the number of days (out of $3 \mathrm{~d}$ reported annually) that each girl consumed cereal, either ready-to-eat or cooked. Cereal food codes were identified using labels in the data. The outcome variables were WHtR (waist and height $(\mathrm{cm}$ ) were measured in all study years except year 1) and total LDL and HDL, cholesterol (mg/dl) derived from $12 \mathrm{~h}$ fasting blood specimens obtained in the morning in study years $1,3,5,7$ and $10^{(23,24)}$. Cut-off values indicating risk for children include (i) $>0.49$ for $\mathrm{WHtR}^{(25)}$; (ii) total cholesterol $>200 \mathrm{mg} / \mathrm{dl}$; (iii) HDL cholesterol $<35 \mathrm{mg} / \mathrm{dl}$; and (iv) LDL cholesterol $>130 \mathrm{mg} / \mathrm{dl}^{(26)}$.

Girls' age was recorded as age at last birthday. Race (black or white) was self-reported. Girls were categorized as being from one- or two-parent households. The highest level of parental education for either parent (an indicator of socio-economic status) was categorized as $\geq 4$ years of college $v .<4$ years. The number of parents in the household was one or two. Age of menarche was calculated as the difference in years between a girl's date of birth and the date when she started having her periods. Physical activity was assessed using the habitual activity questionnaire ${ }^{(27)}$. A physical activity score was computed by multiplying an estimate of 'metabolic equivalents' for the recorded activities by the weekly frequency, duration and fraction of the year during which activities were performed.

\section{Statistical analysis}

The number of days when cereal was eaten in each visit (out of $3 \mathrm{~d}$ possible) was the primary measure of the quantity of cereal consumed. There were two reasons for using the number of days when cereal was eaten as the measure of the quantity consumed. First, gram amounts of the cereal consumed were available only in visit three and after; in earlier visits, participants recorded cereal amounts in a variety of units. Second, the amount of cereal consumed varied within a relatively narrow range for visits when gram amounts of cereal consumed were available, the widest interquartile range was $28-66 \mathrm{~g} / \mathrm{d}^{(28)}$.

Analyses estimated the association of days eating cereal with each outcome variable: WHtR, total cholesterol, HDL cholesterol and LDL cholesterol. Mixed models were used to take full advantage of the longitudinal data ${ }^{(29)}$. Briefly, for each girl, an intercept and slope of days eating cereal with each outcome was estimated (the intercept and slope are random effects, potentially varying across girls), using data for all visits; then the mean slope of days eating cereal with each outcome was computed, averaging across girls and visits. To ensure that the association between cereal consumption and the outcomes did not vary across visits, cereal-by-visit interactions were tested in preliminary models, but the interactions were left out of the final models because they did not approach significance. Mixed models accounted for the correlation among repeated measures within girls and enabled unbiased estimation in the presence of missing data arising from girls' occasional failure to participate in study visits ${ }^{(29,30)}$.

A separate model was estimated for each outcome. Cereal consumption was the predictor of primary interest. All models adjusted for potential confounds of the cereal/ outcome associations, namely age, study site, race, age of menarche, parental education, number of parents in the household, physical activity, average daily energy intake, average daily intake of fibre, fruit, vegetables and milk, respectively, and the number of days eating breakfast (because meal labels were not obtained in the study, breakfast was defined as eating between $5 \mathrm{am}$ and $10 \mathrm{am}$ on weekdays or $5 \mathrm{am}$ and $11 \mathrm{am}$ on weekends, based on previous study ${ }^{(31)}$ ). Parameter estimates, $95 \% \mathrm{CI}$ and hypothesis tests are reported for cereals; the other covariates were 
viewed as adjustment variables (potential confounds of the cereal effects), and therefore estimates for these are not reported but are available from the authors upon request.

Statistical tests compared the lowest level of cereal consumption $(0 \mathrm{~d}$ eating cereal in a visit, or infrequent cereal consumption) with each higher level of cereal consumption. All tests were two-sided; $P<0.05$ was the criterion of statistical significance. Analyses were performed with the SAS statistical software package version 9·1 (SAS Institute, Cary, NC, USA).

\section{Results}

The analysis included the 2371 girls who completed a full $3 \mathrm{~d}$ food diary in at least one study year; of these girls, $98.2 \%$ had food diaries available in two or more study years. Of the 2371 girls included in the analysis, 51.0\% were black, $35 \cdot 1 \%$ had a parent with $\geq 4$ years of college education, $68.5 \%$ were from two-parent households, $37 \cdot 3 \%$ were from the Berkeley site, $36 \cdot 5 \%$ from the Cincinnati site and $26 \cdot 2 \%$ from the Westat/Group Health site. Table 1 shows descriptive statistics for cereal consumption and the outcome variables, by age. Table 2 shows the basic sociodemographic characteristics, physical activity score and nutrition indicators (energy, fruit, vegetable and milk intake) by the average number of days eating cereal (out of $3 \mathrm{~d}$ possible) across all visits.

On average, girls who consumed cereal on $3 \mathrm{~d}$ had significantly lower WHtR than girls who consumed cereal on $0 \mathrm{~d}$ (Table 3). Girls who consumed cereal on 2 or $3 \mathrm{~d}$ had significantly lower total cholesterol than did those who consumed cereal on $0 \mathrm{~d}$, on average. On average, girls who consumed cereal on 1, 2 or $3 \mathrm{~d}$ had significantly lower LDL cholesterol than did those who consumed cereal on $0 \mathrm{~d}$. However, there was no significant association between cereal consumption and HDL cholesterol. These analyses adjusted for the measures of sociodemographic characteristics, physical activity and nutrition shown in Table 2 (as well as the study site, age and number of days eating breakfast). Therefore, the results are interpreted as the estimated association of days eating cereal with each outcome, holding these other factors constant.

The results indicate that adolescent girls who ate cereal more often had lower WHtR, as well as lower total cholesterol and lower LDL cholesterol, even after adjusting for differences in sociodemographic factors, physical activity, nutrition variables and total energy intake. However, it should be noted that none of the mean values fell into the at-risk range for the variables studied in this sample. Earlier study ${ }^{(32)}$ found that cereal consumption was related to BMI; the present study extends that finding to potentially include more powerful predictors of CVD risk and offers suggestive evidence that cereal eating is associated with markers of cardiovascular risk in adolescent girls.

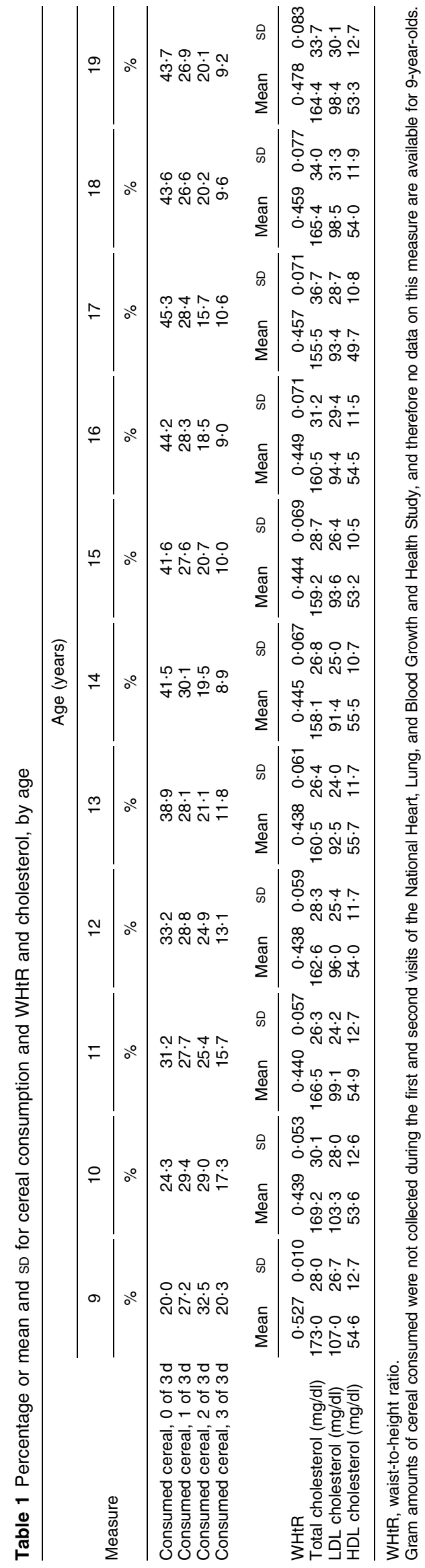


Table 2 Mean and SD of measure or percentage of girls by average number of days eating cereal (out of $3 \mathrm{~d}$ possible) across visits, for selected measures of sociodemographic characteristics, physical activity and nutrition

\begin{tabular}{|c|c|c|c|c|c|c|c|c|}
\hline \multirow[b]{2}{*}{ Measure } & \multicolumn{8}{|c|}{ Average number of days eating cereal (out of $3 \mathrm{~d}$ possible) across visits } \\
\hline & $0 d$ & & $>0-1 d$ & & $>1-2 d$ & & $>2 d$ & \\
\hline Number of girls & 75 & & 1123 & & 991 & & 182 & \\
\hline Black (\%) & $60 \cdot 0$ & & $59 \cdot 5$ & & $44 \cdot 1$ & & $33 \cdot 0$ & \\
\hline \multirow[t]{2}{*}{ College-educated parent (\%) } & $30 \cdot 7$ & & $32 \cdot 0$ & & $35 \cdot 9$ & & $52 \cdot 5$ & \\
\hline & Mean & SD & Mean & SD & Mean & SD & Mean & SD \\
\hline Number of parents in household & $1 \cdot 73$ & 0.45 & $1 \cdot 67$ & 0.47 & 1.69 & 0.46 & $1 \cdot 76$ & 0.43 \\
\hline Physical activity score (MET) & $21 \cdot 1$ & $12 \cdot 6$ & $18 \cdot 3$ & $11 \cdot 4$ & $19 \cdot 6$ & $11 \cdot 5$ & $22 \cdot 5$ & $11 \cdot 8$ \\
\hline \multicolumn{9}{|l|}{ Average daily energy intake } \\
\hline $\mathrm{kJ}$ & 7643 & 1856 & 7753 & 1791 & 8043 & 1797 & 8086 & 1804 \\
\hline kcal & 1827 & 444 & 1853 & 428 & 1922 & 430 & 1933 & 431 \\
\hline Average daily fibre intake $(\mathrm{g})$ & $9 \cdot 7$ & $3 \cdot 0$ & $10 \cdot 9$ & $3 \cdot 0$ & $12 \cdot 1$ & $3 \cdot 3$ & $13 \cdot 1$ & $3 \cdot 7$ \\
\hline \multicolumn{9}{|l|}{ Average daily fruit intake } \\
\hline $\mathrm{kJ}$ & $293 \cdot 7$ & $287 \cdot 0$ & $339 \cdot 3$ & $236 \cdot 4$ & $371 \cdot 1$ & $241 \cdot 8$ & $453 \cdot 1$ & $272 \cdot 0$ \\
\hline kcal & $70 \cdot 2$ & $68 \cdot 6$ & $81 \cdot 1$ & $56 \cdot 5$ & $88 \cdot 7$ & $57 \cdot 8$ & $108 \cdot 3$ & $65 \cdot 0$ \\
\hline \multicolumn{9}{|l|}{ Average daily vegetable intake } \\
\hline kJ & $475 \cdot 3$ & $282 \cdot 4$ & $484 \cdot 5$ & $229 \cdot 3$ & $448 \cdot 5$ & $220 \cdot 9$ & $422 \cdot 2$ & $192 \cdot 5$ \\
\hline kcal & $113 \cdot 6$ & $67 \cdot 5$ & $115 \cdot 8$ & $54 \cdot 8$ & $107 \cdot 2$ & $52 \cdot 8$ & $100 \cdot 9$ & $46 \cdot 0$ \\
\hline \multicolumn{9}{|l|}{ Average daily milk intake } \\
\hline $\mathrm{kJ}$ & $288 \cdot 3$ & $281 \cdot 2$ & $452 \cdot 7$ & $277 \cdot 0$ & 651.9 & $321 \cdot 3$ & $861 \cdot 5$ & 353.5 \\
\hline kcal & $68 \cdot 9$ & $67 \cdot 2$ & $108 \cdot 2$ & $66 \cdot 2$ & $155 \cdot 8$ & $76 \cdot 8$ & $205 \cdot 9$ & $84 \cdot 5$ \\
\hline
\end{tabular}

MET, metabolic equivalent units.

Table 3 Estimated WHtR, total, HDL and LDL cholesterol (mg/dl; $95 \% \mathrm{Cl}$ ) by number of days consuming cerealt

\begin{tabular}{|c|c|c|c|c|c|c|c|c|}
\hline \multirow[b]{3}{*}{ Outcome variable } & \multicolumn{8}{|c|}{ Days consumed cereal (out of $3 \mathrm{~d}$ possible during the $3 \mathrm{~d}$ food diary) } \\
\hline & \multicolumn{2}{|c|}{$0 d$} & \multicolumn{2}{|r|}{$1 d$} & \multicolumn{2}{|c|}{$2 d$} & \multicolumn{2}{|c|}{$3 d$} \\
\hline & Estimate & $95 \% \mathrm{Cl}$ & Estimate & $95 \% \mathrm{Cl}$ & Estimate & $95 \% \mathrm{Cl}$ & Estimate & $95 \% \mathrm{Cl}$ \\
\hline Waist/height ratio & $0 \cdot 450$ & $0.447,0.454$ & $0 \cdot 449$ & $0.444,0.454$ & $0 \cdot 448$ & $0.444,0.453$ & $0 \cdot 440^{*}$ & $0.439,0.449$ \\
\hline Total cholesterol & $162 \cdot 2$ & $159 \cdot 9,164 \cdot 4$ & $161 \cdot 2$ & $158 \cdot 9,163 \cdot 5$ & $160 \cdot 4^{*}$ & $158 \cdot 0,162 \cdot 7$ & $160 \cdot 2^{*}$ & $158 \cdot 6,162 \cdot 8$ \\
\hline HDL cholesterol & $56 \cdot 0$ & $55 \cdot 0,56 \cdot 9$ & $56 \cdot 4$ & $55 \cdot 4,57 \cdot 4$ & $55 \cdot 4$ & $54 \cdot 4,56 \cdot 4$ & $55 \cdot 6$ & $54 \cdot 5,56 \cdot 7$ \\
\hline LDL cholesterol & $94 \cdot 5$ & $92 \cdot 5,96 \cdot 6$ & $93 \cdot 1^{*}$ & $91 \cdot 0,95 \cdot 2$ & $93 \cdot 0^{*}$ & $90 \cdot 8,95 \cdot 2$ & $92 \cdot 5^{\star}$ & $90 \cdot 1,94 \cdot 9$ \\
\hline
\end{tabular}

*Significantly different from $0 \mathrm{~d}$ consuming cereal, $P<0.05$.

tEstimates are from mixed models adjusting for age, study site, race, parental education, age of menarche, number of parents in the household, physical activity level, average daily energy intake, average daily intake of fibre, fruit, vegetables and milk and number of days of eating breakfast. The estimates are interpreted as the expected value of a given outcome variable, averaged over the other covariates, which were centre-coded to enable such interpretation.

\section{Discussion}

The variables that might influence the relationship between cereal consumption and indicators of health have only begun to be studied. Potential mediators of these links include dietary fat levels ${ }^{(18)}$, glycaemic index ${ }^{(33)}$, nutrient fortification $^{(34)}$, consumption of whole grains ${ }^{(35)}$ and breakfast frequency ${ }^{(36)}$. One recent study of adults ${ }^{(37)}$ found significant associations between cereal fibre and several health variables, including lower BMI, smaller waist circumference, and lower cholesterol and glucose levels, suggesting that fibre intake may be one important factor mediating the health effects of cereal consumption. Mozzaffarian et al. ${ }^{(38)}$ found support for this hypothesis with an elderly sample for which cereal fibre consumption was inversely associated with cardiovascular incidents (i.e. stroke, myocardial infarction); individuals in the highest quintile of cereal fibre intake had a $21 \%$ lower risk of cardiovascular events. Other studies have linked whole grains, including oat fibre, to lower cholesterol in adult samples ${ }^{(16,39)}$. Research is needed to better understand the mechanisms by which cereal consumption may lower cholesterol in children and longer-term data will be necessary to determine whether this association continues into adulthood ${ }^{(40)}$.

In the present study, cereal consumption was related to both lower total cholesterol and lower LDL cholesterol in adolescent girls. A previous study with a different sample of children found that higher ready-to-eat cereal consumption was associated with both lower total and LDL cholesterol in boys ${ }^{(41)}$. The mechanisms that might explain this relationship in children are most likely complex and have not been well studied to date. It is possible that the nutrient content of cereal, particularly as related to whole grains, directly impacts the cholesterol levels ${ }^{(42)}$. Another explanation is that the link with cholesterol is 
related to the connection between cereal consumption and physical activity. An earlier study found that children who were high cereal consumers also tended to engage in more physical activity ${ }^{(28)}$, and recent data indicate that higher physical activity is associated with lower cholesterol levels in children and adolescents ${ }^{(43)}$. In a recent review, e.g., five of eight intervention-based studies found significant improvements in at least one lipid/lipoprotein variable in the children who received a physical activity programme, relative to controls ${ }^{(44)}$. Alternatively, previous studies of this cohort have indicated that girls who ate cereal also consumed less fat in their diets, which most likely relates to cholesterol levels ${ }^{(45,46)}$. For example, in a study of 7-year-old children in Finland, serum cholesterol concentrations were found to be lower in those who had diets lower in saturated fat ${ }^{(47)}$, suggesting the possibility of intervention targets. Further study is needed to determine exactly how cereal consumption might influence risk for CVD in children and to develop programmes for reducing cholesterol levels in children at risk.

The relationship between dietary intake, body composition, sexual maturation during adolescence and lipid levels is complex ${ }^{(48)}$. Studies of the effects of diet on HDL cholesterol in children have been less consistent than those on LDL cholesterol ${ }^{(18,49-51)}$, suggesting that dietary changes may have a stronger effect on LDL than HDL cholesterol, as was seen in the present study. Future research exploring interventions to decrease LDL cholesterol and raise HDL cholesterol is needed ${ }^{(52)}$.

There are several limitations to the present study. Due to the large sample size, NGHS had excellent statistical power to detect associations between cereal consumption and health outcomes, and therefore statistically significant differences do not necessarily represent practically large effects. As is typical of epidemiological studies, dietary information was based on self-report and may therefore be subject to recall errors or under-reporting. Because meal labels were not collected in the study, breakfast was defined based on the time of consumption, but these times could include snacks as well as breakfast meals. Finally, although demographically diverse in terms of geography and socio-economic status, the NGHS is not a nationally representative sample of girls. These limitations are offset by several strengths, such as the low attrition rate and the availability of $3 \mathrm{~d}$ of food intake data.

The present study adds to the growing body of evidence indicating that cereal consumption is associated with a number of positive health benefits that should be studied further.

\section{Acknowledgements}

The present study was supported by the General Mills Inc. and by a grant from the National Heart, Lung, and Blood Institute (NHLBI) (HL/DK71122). Drs Franko,
Thompson and Barton are paid consulting fees by General Mills Inc. The present study was also supported by contracts HC55023-26 and cooperative agreements U01-HL-48941-44. Participating NGHS centers included Children's Medical Center, Cincinnati, OH, USA (Stephen $\mathrm{R}$ Daniels, MD, Principal Investigator and John A Morrison, PhD, Co-Investigator); Westat Inc., Rockville, MD, USA (George B Schreiber, ScD, Principal Investigator and Ruth Striegel-Moore, PhD, Co-Investigator); and University of California, Berkeley, CA, USA (Zak I Sabry, PhD, Principal Investigator, Patricia B Crawford, MPH, Dr PH, RD, Co-Investigator); Maryland Medical Research Institute, Baltimore, MD (Bruce A Barton, PhD, Principal Investigator) served as the data coordinating centre. Program Office: NHLBI (Eva Obarzanek, PhD, MPH, RD, Project Officer 1992-2007, Gerald H Payne, MD, Project Officer 1985-1991). The authors have no conflict of interest to declare. D.L.F. and A.M. designed the study and wrote the manuscript; D.R.T. designed the study, analysed the data and wrote the manuscript; and B.A.B. designed the study and analysed the data. Portions of the present manuscript were completed while one of the authors (D.L.F.) was a distinguished research fellow at the Institute for Advanced Study and the School of Psychological Science at La Trobe University, Melbourne, Australia.

\section{References}

1. Albertson AM, Anderson GH, Crockett SJ et al. (2003) Ready-to-eat cereal consumption its relationship with BMI and nutrient intake of children aged 4 to 12 years. J Am Diet Assoc 103, 1613-1619.

2. Cho S, Dietrich M, Brown CJ et al. (2003) The effect of breakfast type on total daily energy intake and body mass index: results from the Third National Health and Nutrition Examination Survey (NHANES III). J Am Coll Nutr 22, 296-302.

3. Kosti RI, Panagiotakos DB, Zampelas A et al. (2008) The association between consumption of breakfast cereals and BMI in schoolchildren aged 12-17 years: the VYRONAS Study. Public Health Nutr 11, 1015-1021.

4. Nicklas TA, O'Neil CE \& Berenson GS (1998) Nutrient contribution of breakfast, secular trends, and the role of ready-to-eat cereals: a review of data from the Bogalusa Heart Study. Am J Clin Nutr 67, Suppl., 757S-763S.

5. Sampson AE, Dixit S, Meyers AF et al. (1995) The nutritional impact of breakfast consumption on the diets of inner-city African-American elementary school children. J Natl Med Assoc 87, 195-202.

6. Williams P (2007) Breakfast and the diets of Australian children and adolescents: an analysis of data from the 1995 National Nutrition Survey. Int J Food Sci Nutr 58, 210-216.

7. Aekplakorn W, Kosulwat V \& Suriyawongpaisal P (2005) Obesity indices and cardiovascular risk factors in Thai adults. Int J Obes (Lond) 12, 1782-1790.

8. Can AS, Bersot TP \& Gonen M (2009) Anthropometric indices and their relationship with cardiometabolic risk factors in a sample of Turkish adults. Public Health Nutr 12, 538-546.

9. Hsieh SD, Muto T, Yoshinaga H et al. (2006) Waist-toheight ratio, a simple and effective predictor for metabolic risk in Japanese men and women. Int Congr Ser 1294, 186-189. 
10. Lin WY, Lee LT, Chen CY et al. (2002) Optimal cut-off values for obesity: using simple anthropometric indices to predict cardiovascular risk factors in Taiwan. Int $J$ Obes Relat Metab Disord 26, 1232-1238.

11. Lee CMY, Huxley RR, Wildman RP et al. (2008) Indices of abdominal obesity are better discriminators of cardiovascular risk factors than BMI: a meta-analysis. I Clin Epidemiol 61, 646-653.

12. Kahn HS, Imperatore G \& Cheng YJ (2005) A populationbased comparison of BMI percentiles and waist-to-height ratio for identifying cardiovascular risk in youth. $J$ Pediatr 146, 482-488.

13. McCarthy HD \& Ashwell M (2006) A study of central fatness using waist-to-height ratios in UK children and adolescents over two decades supports the simple message - 'keep your waist circumference to less than half your height'. Int J Obes (Lond) 30, 988-992.

14. Moran A, Jacobs DR Jr, Steinberger J et al. (2008) Changes in insulin resistance and cardiovascular risk during adolescence. Establishment of differential risk in males and females. Circulation 117, 2361-2368.

15. Kwiterovich PO (2008) Primary and secondary disorders of lipid metabolism in pediatrics. Pediatr Endocrinol Rev 5, Suppl. 2, 727-738.

16. Kelly SA, Summerbell CD, Brynes A et al. (2007) Wholegrain cereals for coronary heart disease. Cochrane Database Syst Rev, issue 2, CD005051.

17. Spiotta RT \& Luma GB (2008) Evaluating obesity and cardiovascular risk factors in children and adolescents. $\mathrm{Am}$ Fam Phys 78, 1052-1058.

18. Gibson S (2003) Micronutrient intakes, micronutrient status and lipid profiles among young people consuming different amounts of breakfast cereals further analysis of data from the National Diet and Nutrition Survey of Young People aged 4 to 18 years. Public Health Nutr 6, 815-820.

19. Nicklas TA, von Duvillard SP \& Berenson GS (2002) Tracking of serum lipids and lipoproteins from childhood to dyslipidemia in adults: the Bogalusa Heart Study. Int $J$ Sports Med 23, Suppl. 1, S39-S43.

20. NHLBI Study Group (1992) Obesity and cardiovascular disease risk factors in black and white girls: NHLBI Growth and Health Study. Am J Public Health 82, 1613-1620.

21. Crawford PB, Obarzanek E, Morrison J et al. (1994) Comparative advantage of 3-day food records to 24-hour recall or 5-day food frequency validated by observation of 9- and 10-year-old girls. J Am Diet Assoc 94, 626-630.

22. Schakel GH, Sievert YA \& Buzzard IM (1988) Sources of data for developing and maintaining a nutrient database. $J$ Am Diet Assoc 88, 1268-1271.

23. Delong DM, DeLong ER, Wood PD et al. (1986) A comparison of methods for the estimation of plasma lowand very low-density lipoprotein cholesterol. The Lipid Research Clinics Prevalence Study. J Am Med Assoc 256, 2372-2377.

24. Friedewald WT, Levy RI \& Fredrickson DS (1972) Estimation of the concentration of low-density lipoprotein cholesterol in plasma, without use of the preparative ultracentrifuge. Clin Chem 18, 499-502.

25. Maffeis C, Banzato C \& Talamini G (2008) Obesity Study Group of the Italian Society of Pediatric Endocrinology and Diabetology. Waist-to-height ratio, a useful index to identify high metabolic risk in overweight children. J Pediatr 152, 207-213.

26. Lee JM, Gebremariam A, Card-Higginson P et al. (2009) Poor performance of body mass index as a marker for hypercholesterolemia in children and adolescents. Arch Pediatr Adolesc Med 163, 716-723.

27. Ku LC, Shapiro LR, Crawford PB et al. (1981) Body composition and physical activity in 8-year-old children. Am J Clin Nutr 34, 2770-2775.
28. Albertson AM, Thompson D, Franko DL et al. (2009) Prospective associations among cereal intake in childhood and adiposity, lipid level, and physical activity during late adolescence. J Am Diet Assoc 109, 1775-1780.

29. Verbeke G \& Molenberghs G (2000) Linear Mixed Models for Longitudinal Data. New York: Springer Verlag.

30. Beunckens C, Molenberghs G \& Kenward MG (2005) Direct likelihood analysis versus simple forms of imputation for missing data in randomized clinical trials. Clin Trials 2, 379-386.

31. Affenito SG, Thompson DR, Barton BA et al. (2005) Breakfast consumption by African-American and white adolescent girls correlates positively with calcium and fiber intake and negatively with body mass index. $J$ Am Diet Assoc 105, 938-945.

32. Barton BA, Eldridge AL, Thompson D et al. (2005) The relationship of breakfast and cereal consumption to nutrient intake and body mass index: the National Heart, Lung and Blood Institute Growth and Health Study. J Am Diet Assoc 105, 1383-1389.

33. Backhouse SH, Williams C, Stevenson E et al. (2007) Effects of the glycemic index of breakfast on metabolic responses to brisk walking in females. Eur J Clin Nutr 61, 590-596.

34. Rampersaud GC, Pereira MA, Girard BL et al. (2005) Breakfast habits, nutritional status, body weight, and academic performance in children and adolescents. $J \mathrm{Am}$ Diet Assoc 105, 743-760.

35. Kochar J, Djousse L \& Gaziano JM (2007) Breakfast cereals and risk of type 2 diabetes in the Physicians' Health Study I. Obesity (Silver Spring) 15, 3039-3044.

36. Timlin MT, Pereira MA, Story M et al. (2008) Breakfast eating and weight change in a 5 -year prospective analysis of adolescents: Project EAT (Eating Among Teens). Pediatrics 121, e638-e645.

37. Newby PK, Maras J, Bakun P et al. (2007) Intake of whole grains, refined grains, and cereal fiber measured with 7-d diet records and associations with risk factors for chronic diseases. Am J Clin Nutr 86, 1745-1753.

38. Mozaffarian D, Kumanyika SK, Lemaitre RN et al. (2003) Cereal, fruit, and vegetable fiber intake and the risk of cardiovascular disease in elderly individuals. JAMA 289, 1659-1666.

39. Davy BM, Davy KP, Ho RC et al. (2002) High-fiber oat cereal compared with wheat cereal consumption favourably alters LDL cholesterol subclass and particle numbers in middle-aged and older men. Am J Clin Nutr 76, 351-358.

40. Srinivasan SR, Frontini MG, Xu J et al. (2006) Utility of childhood non-high-density lipoprotein cholesterol levels in predicting adult dyslipidemia and other cardiovascular risks: The Bogalusa Heart Study. Pediatrics 118, 201-206.

41. Albertson AM, Affenito SG, Bauserman R et al. (2009) The relationship of ready-to-eat cereal consumption to nutrient intake, blood lipids, and body mass index of children as they age through adolescence. J Am Diet Assoc 109, $1557-1565$.

42. Kelly SA, Summerbell CD, Brynes A et al. (2007) Wholegrain cereals for coronary heart disease. Cochrane Database Syst Rev, issue 2, CD005051.

43. Owen CG, Nightingale CM, Rudnicka AR et al. (2010) Physical activity, obesity and cardiometabolic risk factors in 9- to 10-year-old UK children of white European, South Asian and black African-Caribbean origin: the Child Heart And health Study in England (CHASE). Diabetologia 53, $1620-1630$.

44. Janssen I \& Leblanc AG (2010) Systematic review of the health benefits of physical activity and fitness in schoolaged children and youth. Int J Behav Nutr Phys Act 7, 40.

45. Barton BA, Eldridge AL, Thompson D et al. (2005) The relationship of breakfast and cereal consumption to nutrient intake and body mass index: the National Heart, 
Lung and Blood Institute Growth and Health Study. J Am Diet Assoc 105, 1383-1389.

46. Albertson AM, Thompson D, Franko DL et al. (2008) Consumption of ready-to-eat cereal is associated with positive health outcomes: evidence from the National Heart, Lung, and Blood Institute Growth and Health Study. Nutr Res 28, 744-752.

47. Räsänen M, Lehtinen JC, Niinikoski H et al. (2002) Dietary patterns and nutrient intakes of 7-year-old children taking part in an atherosclerosis prevention project in Finland. J Am Diet Assoc 102, 518-524.

48. Kwiterovich PO Jr, Barton BA, McMahon RP et al. (1997) Effects of diet and sexual maturation on low-density lipoprotein cholesterol during puberty; the Dietary Intervention Study in Children (DISC). Circulation 96, 2526-2533.
49. The Writing Group for the DISC Collaborative Research Group (1995) Efficacy and safety of lowering dietary intake of fat and cholesterol in children with elevated low-density lipoprotein cholesterol. The Dietary Intervention Study in Children (DISC). J Am Med Assoc 273, 1429-1435.

50. Mietus-Synder M, Baker AL \& Neufeld EJ (1993) Effects of nutritional counselling on lipoprotein levels in a pediatric clinic. Am J Disord Child 147, 378-381.

51. Polonsky SM, Bellet PS \& Specher DL (1993) Primary hyperlipidemia in a pediatric population: classification and effect of dietary treatment. Pediatrics 91, 92-96.

52. Newfield RS, Dewan AK \& Jain S (2008) Dyslipidemia in children with type 2 diabetes vs. obesity. Pediatr Diabet 9, 115-121. 